\title{
Synthesis of Poly(styrene-acrylates-acrylic acid) Microspheres and Their Chemical Composition towards Colloidal Crystal Films
}

\author{
Luis A. Ríos-Osuna, ${ }^{1}$ Angel Licea-Claverie, ${ }^{1}$ \\ F. Paraguay-Delgado, ${ }^{2}$ and Norma A. Cortez-Lemus ${ }^{1}$ \\ ${ }^{1}$ Centro de Graduados e Investigación en Química, Instituto Tecnológico de Tijuana, A.P. 1166, 22000 Tijuana, BC, Mexico \\ ${ }^{2}$ Centro de Investigación en Materiales Avanzados S. C. (CIMAV), Miguel de Cervantes 120, 31109 Chihuahua, CHIH, Mexico
}

Correspondence should be addressed to Norma A. Cortez-Lemus; ncortez@tectijuana.mx

Received 28 August 2016; Revised 23 October 2016; Accepted 17 November 2016

Academic Editor: Önder Pekcan

Copyright ( $) 2016$ Luis A. Ríos-Osuna et al. This is an open access article distributed under the Creative Commons Attribution License, which permits unrestricted use, distribution, and reproduction in any medium, provided the original work is properly cited.

In this paper, polystyrene colloidal microspheres have been prepared using hexyl acrylate (HA), ethylhexyl acrylate (EHA), isooctyl acrylate (IOA), butyl acrylate (BA), or isobutyl acrylate (IBA) as comonomers. Microspheres with diameters from 212 to $332 \mathrm{~nm}$ and with a polystyrene content of $65-78 \%$ were prepared. The particles prepared in this work do not present the typical coreshell structure; as a consequence, DSC analysis showed that the microspheres exhibited only one $T_{g}$. TEM images show that the particles with comonomer content below $\sim 30 \%$ were spherical and regular. Microspheres containing comonomer between 21 to $25 \%$ produced the less brittle films showing very iridescent colors. The films prepared from microspheres containing hexyl, ethylhexyl, and isooctyl acrylate as comonomers are firmly attached to the substrate due to their adhesive properties. The large decrease of the fragility observed in these films makes them much more attractive materials in sensing applications.

\section{Introduction}

Colloidal polymeric microspheres have been extensively studied as candidates for construction of photonic crystals used in many optical applications, such as sensors, bioassays, color displays, solar energy, and lasers [1,2]. For most of these applications, size control, narrow size distribution, surface electrical properties, morphology, copolymer composition, and cost become important [3-10]. Over several decades, a rich variety of approaches have been described for producing such well-controlled polymer microspheres such as suspension, emulsion, dispersion, and precipitation polymerization [11]. Moreover, many of these articles have focused their research on the preparation of polymeric crystal films from self-assembly of polymeric microspheres. The polystyrene and polyacrylate-based microspheres are kinds of polymeric materials being widely used due to their excellent properties [12, 13]; for instance, colloidal crystal films prepared using poly(styrene-co-methyl methacrylate$c o$-acrylic acid) poly(Sty- $b$-MMA- $b$-AA) microspheres with a core-shell structure have been extensively investigated [14]. Wang et al. [9] prepared microspheres of poly(Sty- $b$-MMA$b$-AA). The authors demonstrated the core-shell morphology with polystyrene in the core and elastomeric poly(methyl methacrylate- $b$-acrylic acid) shell using X-ray photoelectron spectroscopy (XPS). They estimated the chemical composition distribution from the surface to the interior of the microsphere. Nevertheless, most of the researches on the synthesis of polymeric particles to prepare photonic films do not provide information about the chemical composition of the microsphere. Most of the literature provides information only about the molar ratios of the monomers involved in the formulation. Zhang et al. [13] synthesized microspheres using emulsion copolymerization of styrene with methyl methacrylate and acrylic acid. The correlation between comonomers in the feed and in the microsphere was 
investigated by ${ }^{1} \mathrm{H}-\mathrm{NMR}$. The molar ratio of polystyrene in the microsphere was 90 to $96 \%$, estimated by ${ }^{1} \mathrm{H}-\mathrm{NMR}$. The authors found that acrylic acid content always was lower than the feed values. Moreover, microspheres with higher contents of acrylic acid led to a reduction of the average particle size. On the other hand, several authors have demonstrated that the carboxyl-rich surface of the microspheres enhanced the formation of hydrogen bonds among the carboxyl group of microspheres improving properties like colloidal stability as well as mechanical properties of the film prepared [13-19]. The hard polystyrene core and soft shell structure has been demonstrated using TEM with chemical staining since the core and the shell densities are very similar [11, 20-22].

Although there are many studies devoted to the preparation and characterization of polymeric microspheres based on polystyrene and their use to prepare colloidal crystals, to the best of our knowledge, in the literature no studies have been reported on the preparation of microspheres based on polystyrene using hexyl, ethylhexyl, and isooctyl acrylate as comonomers. Because these polymers well-known as "high alkyl acrylates" are widely used as adhesives [23], their incorporation in the copolymer allows the fabrication of iridescent films which adhere strongly to the substrate, due to the adhesive properties of these polymers. The molar ratio of each component was estimated in all synthesized microspheres. Furthermore, the most optimal ratio between styrene and acrylate to obtain less brittle films with photonic stop-bands in the visible light region was investigated.

\section{Materials and Methods}

2.1. Materials. Styrene (Sty), hexyl acrylate (HA), ethylhexyl acrylate (EHA), isooctyl acrylate (IOA), butyl acrylate (BA), and isobutyl acrylate (IBA) reagents were purchased from Sigma-Aldrich and were used after inhibitor was removed by passing the monomers through a column of inhibitor remover (Sigma-Aldrich). Acrylic acid (AA, Sigma-Aldrich) was used as ionic comonomer without further purification. Sodium dodecylbenzenesulfonate (SDBS), ammonium persulfate (APS), and $\mathrm{NaHCO}_{3}$ were obtained from SigmaAldrich and used as received. The microscope slides used as substrates were cleaned by soaking in a standard piranha solution for 10 minutes at $40^{\circ} \mathrm{C}$; subsequently they were rinsed with deionized water and dried by simple evaporation.

\subsection{Synthesis of Microspheres of Polystyrene with Different} Acrylate as Comonomers. Microspheres of poly(styrene-coethyl hexyl acrylate-co-acrylic acid) (poly(Sty-co-EHA-coAA)) were prepared via batch emulsion polymerization (see Scheme 1). Briefly, styrene $(19.97 \mathrm{mmol})$, ethylhexyl acrylate $(2.434 \mathrm{mmol})$, and acrylic acid $(2.765 \mathrm{mmol})$ were dispersed in $15 \mathrm{~mL}$ deionized water in which $2.260 \mu$ moles of sodium dodecyl-benzene sulfonate and $1.250 \mu$ moles of $\mathrm{NaHCO}_{3}$ were dissolved. The mixture was poured in a Schlenk flask and deoxygenated in a nitrogen atmosphere for $20 \mathrm{~min}$ at room temperature with constant vigorous stirring. The reaction mixture was heated at $70^{\circ} \mathrm{C}$ for $0.5 \mathrm{~h}$. Finally $0.420 \mathrm{mmol}$ of APS was added, to allow the reaction to proceed for $8 \mathrm{~h}$. Upon completion of polymerization, the turbid dispersion was purified by ultracentrifugation several times at $12000 \mathrm{rpm}$ with addition of deionized water to remove unreacted initiator and monomer each time.

\subsection{Characterization of the Synthesized Microspheres}

2.3.1. Differential Scanning Calorimetry (DSC). The microspheres were purified and dried in an oven at $70^{\circ} \mathrm{C}$ for $24 \mathrm{~h}$ to constant weight and their glass transition temperatures $\left(T_{q}\right)$ were obtained on a TA Instrument modulated DSC 2920. Analyses were performed in a helium atmosphere. Samples of 8-12 $\mathrm{mg}$ were heated over aluminum pans at a heating rate of $5^{\circ} \mathrm{C} / \mathrm{min}$ in the temperature range from 0 to $100^{\circ} \mathrm{C}$.

2.3.2. DLS Analysis. Dynamic light scattering (DLS) measurements were performed using diluted samples of microspheres at $25^{\circ} \mathrm{C}$ using a Nano-ZS Nanosizer (Malvern Instruments, ZEN 3690). The instrument was equipped with a helium neon laser (emission wavelength of $633 \mathrm{~nm}$ ) with a size detection range of $0.6 \mathrm{~nm}-5 \mu \mathrm{m}$. DLS experiments were performed at a scattering angle of $90^{\circ}$, and the samples were equilibrated for $2 \mathrm{~min}$ before data collection. The intensityaverage hydrodynamic diameter $\left(D_{h}\right)$ and polydispersity index (PDI) were calculated using Malvern Instruments dispersion technology software based on CONTIN analysis and the Stokes-Einstein equation for spheres.

2.3.3. ${ }^{1} \mathrm{H}$-NMR Analysis of the Microspheres. ${ }^{1} \mathrm{H}$-NMR spectra were collected on Bruker $(400 \mathrm{MHz})$ spectrometer and are reported in ppm using TMS as the internal standard. The solvent used was deuterated chloroform, $\mathrm{CDCl}_{3}$, for all of the samples. Once purified, the microspheres were completely dried in an oven at $70^{\circ} \mathrm{C}$ for $24 \mathrm{~h}$ before analysis.

2.3.4. TEM and SEM Analysis. Morphology of the microspheres on surfaces was assessed by microscopy techniques: scanning electron microscopy (SEM) with a JEOL 4500 JIB and transmission electron microscopy (TEM) using a JEOL JSM 2010F and JEOL 220FS TEM and STEM microscope working at $200 \mathrm{kV}$. For analysis by SEM few drops of the microsphere dispersion were placed on slides and allowed to dry on their own. Afterwards, a thin film of gold was deposited over the slide surface. For TEM analysis, the sample was prepared by placing a few drops of diluted microsphere dispersion on lacy carbon copper grids and allowed to dry.

2.3.5. Optical Response. To determine the optical response of the photonic crystal, a glass fiber was coupled to the USB4000 spectrometer from Ocean Optics, which analyzes the light reflected by the photonic crystal.

2.3.6. Growing Photonic Crystals Films. The dip-coating method was used for growing photonic crystals of selfassembled colloidal particles. The procedure begins by redispersing the particles by sonication in water for 20 minutes and then placing the dispersion in a constant temperature chamber at 30 or $40^{\circ} \mathrm{C}$; next the substrate was immersed in the particles solution, and it was extracted from the solution at a constant rate of seven microns per second. 


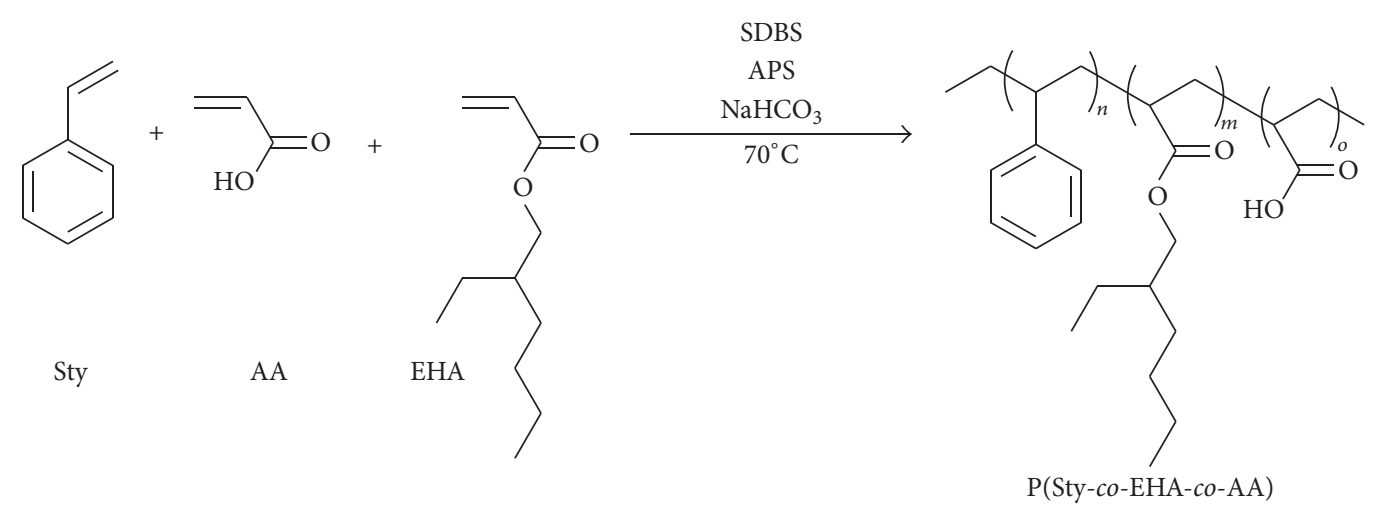

(a)

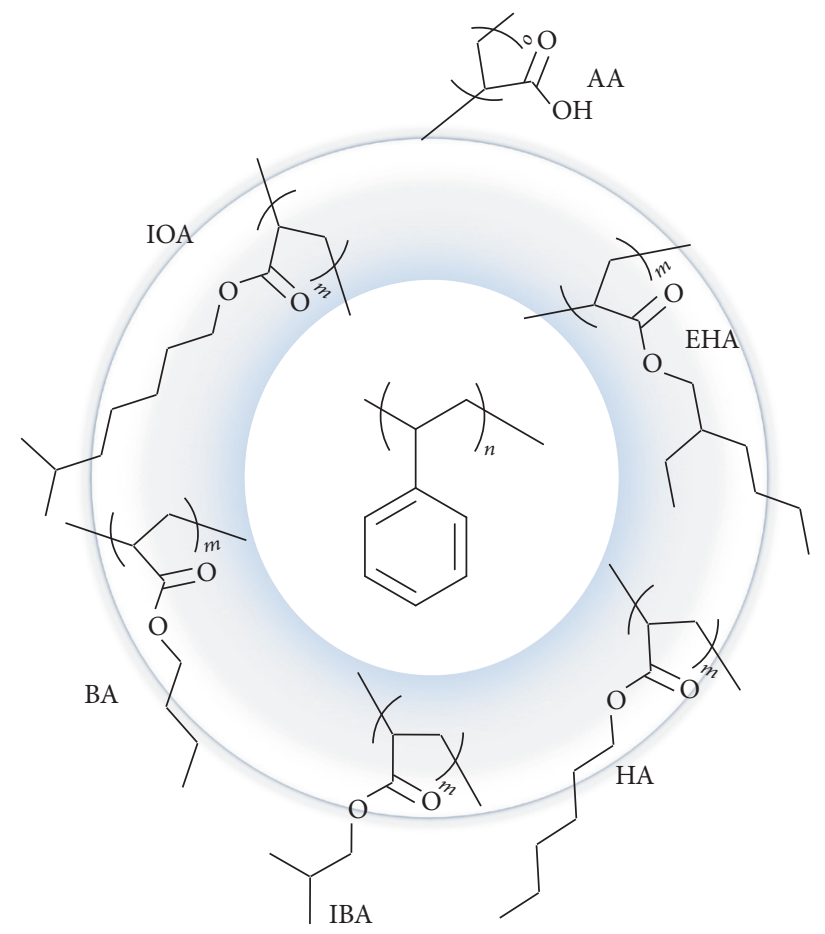

(b)

Scheme 1: (a) Synthesis route for the synthesis of microspheres of poly(styrene-co-ethylhexyl acrylate-co-acrylic acid). (b) Chemical structure of comonomers used for the preparation of microspheres.

\section{Results and Discussion}

In the present study, the first goal was to obtain a series of new polymeric microspheres in one step via batch emulsion polymerization of styrene with several acrylates such as hexyl acrylate, ethylhexyl acrylate, isooctyl acrylate, butyl acrylate, or isobutyl acrylate as comonomers. The resulting polymeric microspheres must be adequate to self-assemble in films that reflect bright colors.

Additionally, the ratio of each comonomer was estimated as well as the effect of the content of nonionic comonomer in the morphology of the microsphere and the self-assembly of colloidal crystal films.
3.1. Study of the Chemical Composition of the Microsphere Using Different Comonomers. In this study it was demonstrated that the incorporation of nonionic acrylates in the microspheres was higher than in the formulation for all the cases. Microspheres with 18 to $39 \%$ of IOA (see Table 1 , entries 1-5) were prepared. It can be observed in Table 1 that the IOA content was approximately $40 \%$ higher in the microsphere than in the feed (estimated by ${ }^{1} \mathrm{H}-\mathrm{NMR}$ ). The hydrodynamic diameter of the microspheres is in the range of 230 to $320 \mathrm{~nm}$ (using DLS), although no clear trend for the resulting particle sizes as an effect of the IOA concentration in the reaction was observed. Microspheres with 16 to $32 \%$ of EHA were also prepared (entries 6-10). Since the 


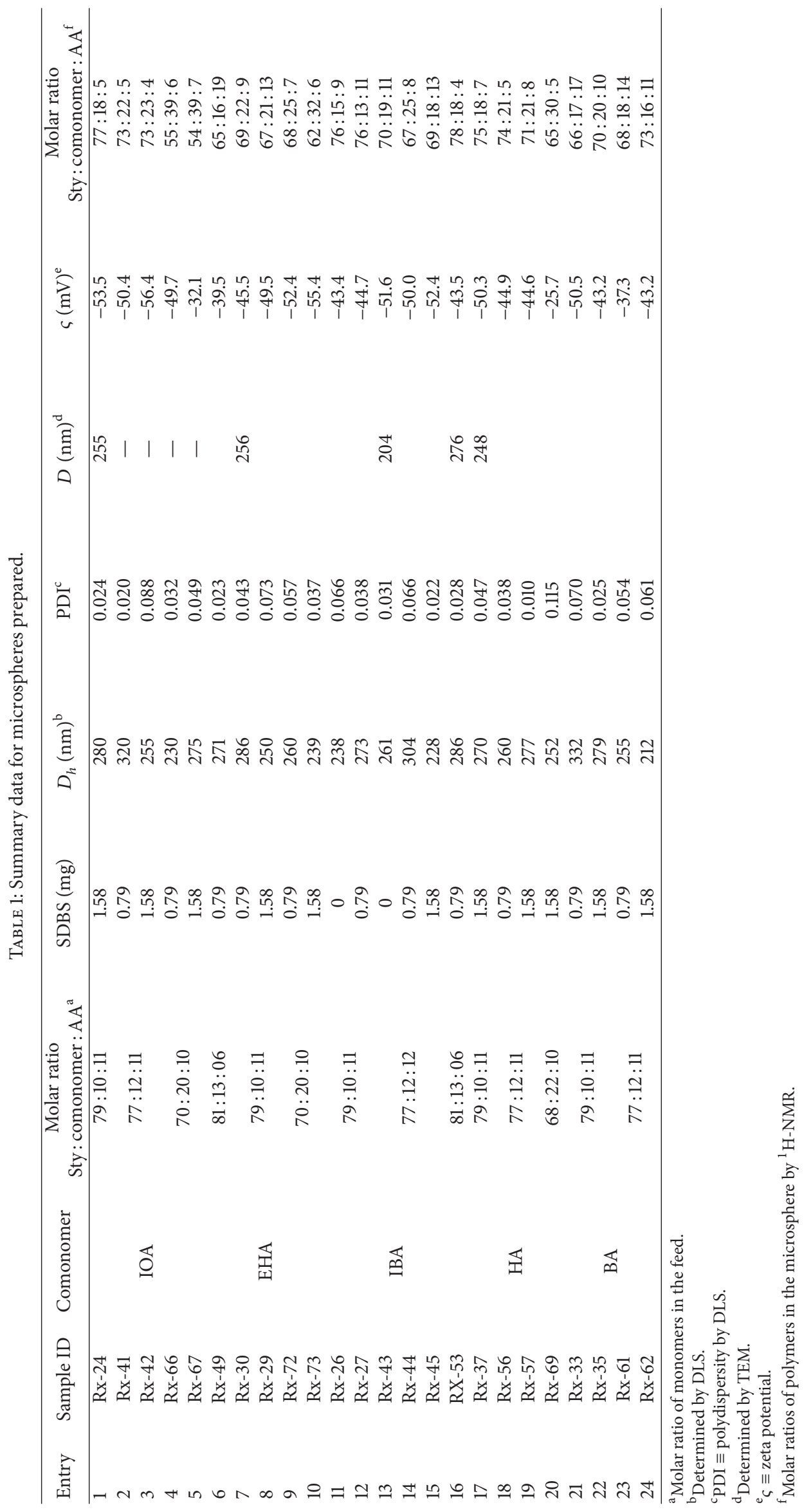




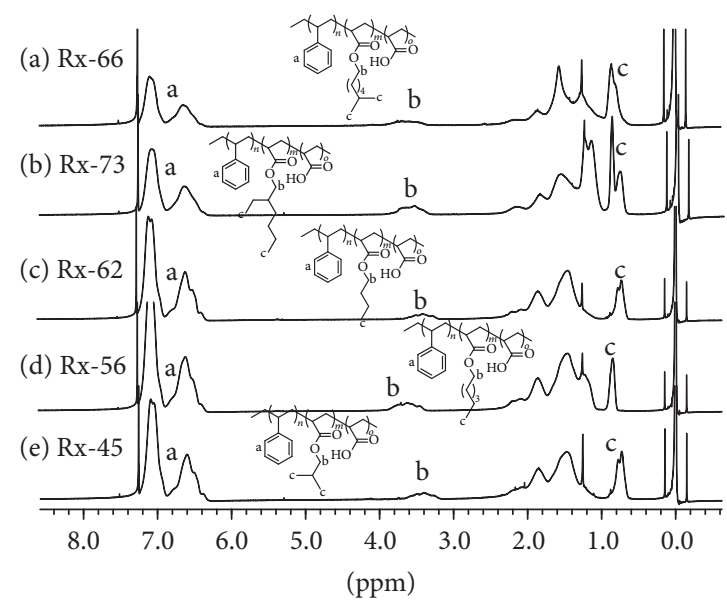

FIgURE 1: ${ }^{1} \mathrm{H}$-NMR spectra of microspheres prepared in this study containing different comonomers. (a) Poly(Sty-co-IOA-co-AA). (b) Poly(Sty-co-EHA-co-AA). (c) Poly(Sty-co-BA-co-AA). (d) Poly(Sty-co-HA-co-AA). (e) Poly(Sty-co-IBA-co-AA).

monomer and initiator concentrations remain unchanged, the increase in the concentration of surfactant leads to the formation of a larger number of micelles (particles) which resulted in the formation of slightly smaller particles. A clear reduction in the hydrodynamic diameter of the microspheres was observed increasing the amount of surfactant: 286 to $250 \mathrm{~nm}$ (entries 7 and 8) and 260 to $239 \mathrm{~nm}$ (entries 9 and 10), respectively. Additionally, it was estimated that the IBA content in the microspheres was 15 to $25 \%$ (estimated by ${ }^{1} \mathrm{H}$ NMR). The hydrodynamic diameter of these microspheres was in the range of 228 to $304 \mathrm{~nm}$. Also, Table 1 shows two reactions in which no surfactant was used in the formulation, and the sizes of the microspheres were very similar to those made using surfactant (entry 11, Rx-26, $238 \mathrm{~nm}$, and entry 13, Rx-43, $261 \mathrm{~nm}$ ). Microspheres with 18 to $30 \%$ of HA were prepared (entries 17-20). In these reactions the decrease in particle size was also noted when increasing amount of surfactant used in the formulation: 270 to $260 \mathrm{~nm}$ (entries 17 and 18) and 277 to $252 \mathrm{~nm}$ (entries 19 and 20), respectively. Regarding the microspheres prepared with BA, (entries 2124) they contain 16 to $20 \%$ of this comonomer. It is also noted that the hydrodynamic diameter of the microsphere decreases with increasing concentration of surfactant: 332 to $279 \mathrm{~nm}$ (entries 21 and 22) and 255 to $212 \mathrm{~nm}$ (entries 23 and $24)$, respectively. It is worth noting that the content of acrylic acid in the microspheres is equal to or greater that in the feed, for instance, 11 to $14 \%$ of acrylic acid for $\mathrm{Rx}-23$.

Figure 1 shows ${ }^{1} \mathrm{H}$-NMR spectra of polymeric microspheres showing specifically the most characteristic signals of each monomer unit. The peaks around 6.72 to $7.22 \mathrm{ppm}$ were attributed to the five aromatic hydrogens of styrene (peaks "a"). The peaks "b" around 3.2 to $3.8 \mathrm{ppm}$ (acrylate comonomer) corresponds to the two hydrogens of methylene located next to the ester group. The peaks " $c$ " at a chemical shift around 0.68 to $0.96 \mathrm{ppm}$ was assigned to the hydrogens of the methyl groups of the acrylates (one or two methyl groups in the case of IBA or IOA). Reviewing the literature, there are few studies that estimate the content of the ionic comonomer by ${ }^{1} \mathrm{H}-\mathrm{NMR}$ and the estimation is usually achieved by titration of the ionic groups $[13,14,16]$. It can be observed (Figure 1) that the peaks of the hydrogens corresponded to the acrylic acid that are completely overlapped in the region of $2.5 \mathrm{ppm}$ in the spectrum. Therefore, to calculate the amount of acrylic acid using ${ }^{1} \mathrm{H}-\mathrm{NMR}$, the next methodology was used. As an example reaction 56 (entry 18) is described: the peak corresponded to the aromatic hydrogens of styrene integrated to five (signal " $a$," 6.72-7.22 ppm, 1 per hydrogen); the methylene peak of the hexyl acrylate (signal "b," 3.2-3.8 ppm) integrated to 0.56 (0.18 per hydrogen); finally, the value of integration for the range between 0.6 and $2.6 \mathrm{ppm}$, where methylenes, methines, and methyl-groups from the three monomers are found is 7.12, which corresponded to three hydrogens for styrene, 14 hydrogens for hexyl acrylate, and three hydrogens for acrylic acid. The final molar ratio for this copolymer 56 is calculated to be $74: 21: 5$ for Sty:HA:AA. A representative ${ }^{1} \mathrm{H}-\mathrm{NMR}$ spectrum of Rx-56 is shown in Figure S1 (see Supplementary Material available online at http://dx.doi.org/10.1155/2016/4527526).

As discussed above, the size range of the microspheres prepared in this work is very narrow (212 to $332 \mathrm{~nm}$, by DLS) considering the different reaction conditions and the use of different comonomers. This could be explained due to the fact that acrylic acid is used in almost constant concentration for all reactions. The AA is the hydrophilic monomer in this formulation and has a high affinity with the initiator APS. In this way, the oligomeric radicals of AA are produced and its concentration is maintained practically constant in almost all reactions.

3.2. Zeta Potential Analysis (ৎ) for the Microspheres. Zeta potential $(\varsigma)$ is a crucial physicochemical parameter the stability of colloidal dispersion [24]. Negative zeta potential values shown in Table 1 indicate the presence of microspheres with high colloidal stability $(-25$ to $-56.4 \mathrm{mV})$. No substantial variation in zeta potential was noted when the concentration of the AA was increased slightly. 


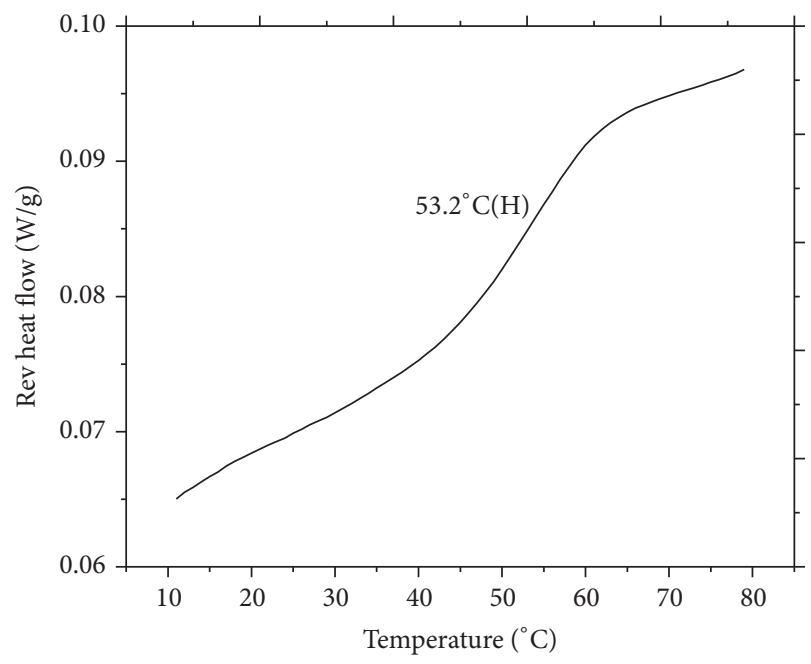

(a)

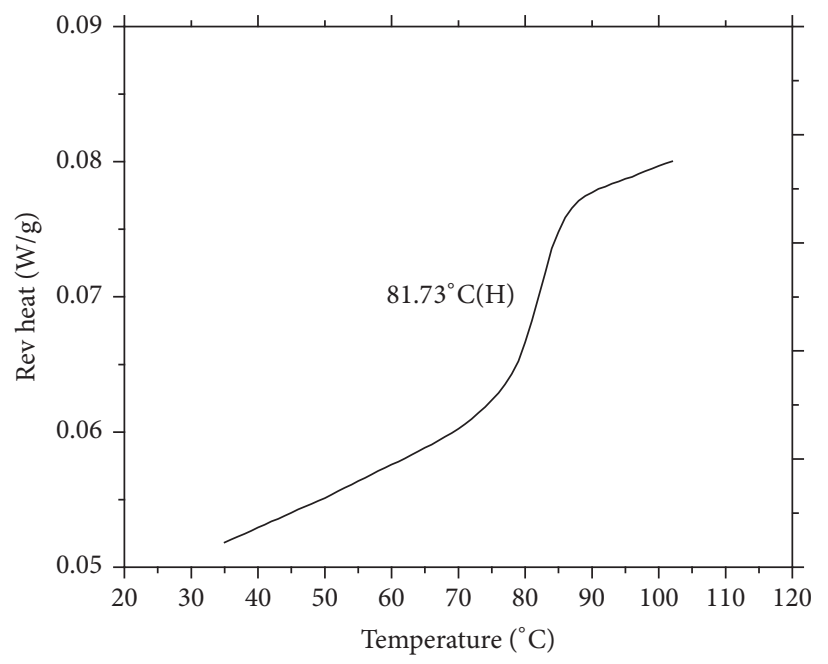

(b)

FiguRE 2: Thermograms by DSC of microspheres of (a) Rx-29 containing poly(ethyl hexyl acrylate) (10\% molar ratio) and (b) Rx-33 containing poly(butyl acrylate) (17\%).

TABLE 2: Glass transition temperature $\left(T_{g}\right)$ for prepared microspheres.

\begin{tabular}{|c|c|c|c|c|}
\hline Sample ID & Copolymer & $\begin{array}{c}\text { Molar ratio } \\
\text { Sty : acrylate: } \mathrm{AA}^{\mathrm{a}}\end{array}$ & $\begin{array}{c}\text { Total acrylate in } \\
\text { the copolymer }(\%)\end{array}$ & $T_{g}{ }^{b}\left({ }^{\circ} \mathrm{C}\right)$ \\
\hline $\mathrm{Rx}-29$ & Sty: EHA : AA & $67: 21: 13$ & 21 & 53.2 \\
\hline $\mathrm{Rx}-73$ & Sty: EHA : AA & $68: 25: 7$ & 32 & 30.5 \\
\hline $\mathrm{Rx}-72$ & Sty: EHA : AA & $64: 28: 8$ & 36 & 25.2 \\
\hline $\mathrm{Rx}-69$ & Sty: HA : AA & $65: 30: 5$ & 35 & 37.3 \\
\hline $\mathrm{Rx}-33$ & Sty: BA : AA & $66: 17: 17$ & 34 & 81.7 \\
\hline $\mathrm{Rx}-35$ & Sty: BA : AA & $70: 20: 10$ & 30 & 80.6 \\
\hline
\end{tabular}

${ }^{\mathrm{a}}$ Molar ratios of monomers in the microsphere by ${ }^{1} \mathrm{H}$ NMR.

${ }^{\mathrm{b}}$ By DSC.

3.3. Glass Transition Temperature $\left(T_{g}\right)$ for Prepared Microspheres. Since the content of acrylates in many of the prepared microspheres of this study is high (according to information obtained by ${ }^{1} \mathrm{H}-\mathrm{NMR}$ ), the resulting $T_{g}$ is low, resulting in coalescence of the microspheres and therefore, producing transparent films (e.g., Rx-66, Rx-67, Rx-69, Rx72, and Rx-73; Table 1).

In the case of DSC analysis (Table 2, Figure 2), only the presence of one $T_{g}$ was observed in all microspheres analyzed. Based in these results, the microspheres do not present the core-shell structure which is indicative when two or more transition phases are detected. As can be seen in Table 2, $T_{g}$ is totally related to the total chemical composition of the polymers in the microsphere (polystyrene $T_{g}=\sim 100^{\circ} \mathrm{C}$ ). Increasing the molar ratio of ethyl hexyl acrylate in the microspheres, $T_{g}$ decreases. As an example, when the molar ratio of EHA in the microsphere reaches $10, T_{g}$ is $53.2^{\circ} \mathrm{C}$, and when the ratio of EHA increased to $28, T_{g}$ drops to $25.2^{\circ} \mathrm{C}$. For the reaction $\mathrm{Rx}-69, \mathrm{~T}_{g}$ is $37.3^{\circ} \mathrm{C}$, when the ratio of $\mathrm{HA}$ is 30 . This value is totally reasonable since $T_{g}$ for EHA is lower than for the HA (poly(ethyl hexyl acrylate) $T_{g}=-65^{\circ} \mathrm{C}$; poly(ethyl hexyl acrylate $T_{g}=-58^{\circ} \mathrm{C}$ ). The microspheres with BA show higher $T_{g}$ (poly(butyl acrylate) $T_{g}=-45^{\circ} \mathrm{C}$ ). For reaction $\mathrm{Rx}-$ 33 , when the ratio of $\mathrm{BA}$ is $17 \mathrm{~T}_{\mathrm{g}}$ is $81.7^{\circ} \mathrm{C}$; but when the ratio of BA increased to $20 T_{g}$ is $80.6^{\circ} \mathrm{C}$. The results of $T_{g}$ provide a good indication that most of the microspheres prepared in this study are suitable to obtain films with iridescent colors.

3.4. Analysis of the Microspheres by TEM and SEM. Figure 3 shows electron microscope micrographs of as synthesized colloidal polystyrene based microspheres. Those particles are monodisperse and the apparent contacts could be due to the slight electron radiation damage to these soft materials and it could be modified and stick among the microspheres; they show uniform smooth surface over all. According to the images, it was not possible to confirm the core-shell structure because the polymers are chemically similar and any interface inside them is indistinguishable. However, there are some works in which the core-shell structure is demonstrated by TEM with or without chemical staining [22]. Microspheres with uneven surface contour were also observed in one case (Figure 3(i)). Hoshino et al. [16] report that an increase in 


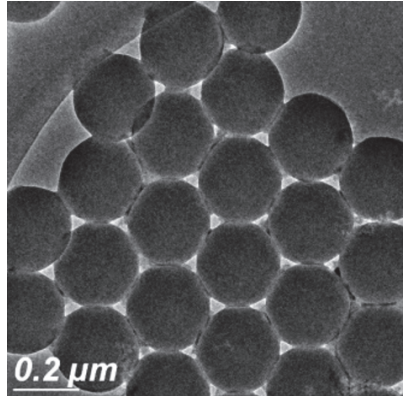

(a)

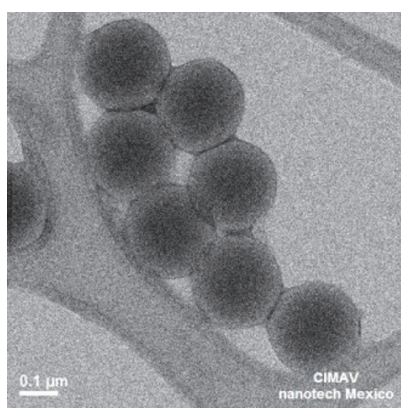

(e)

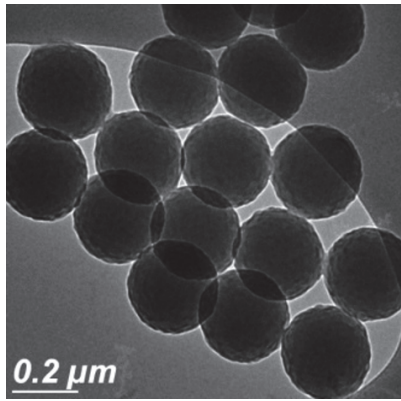

(i)

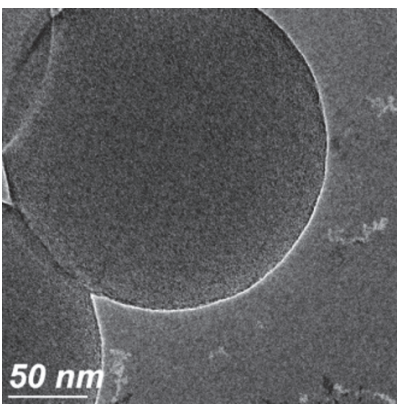

(b)

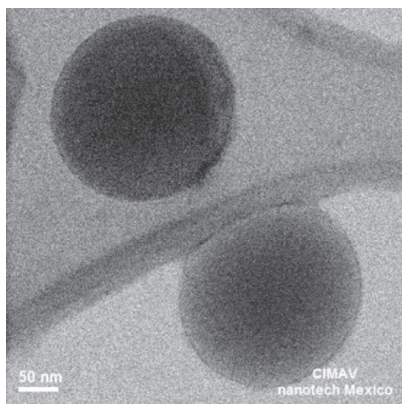

(f)

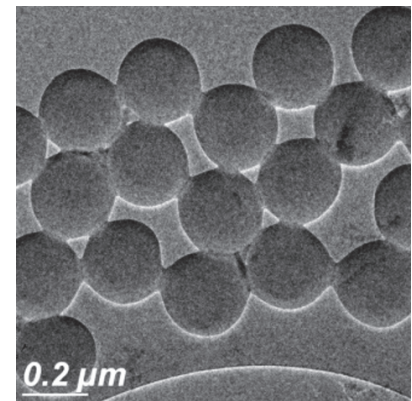

(c)

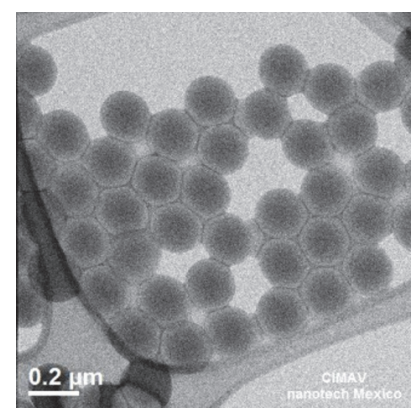

(g)

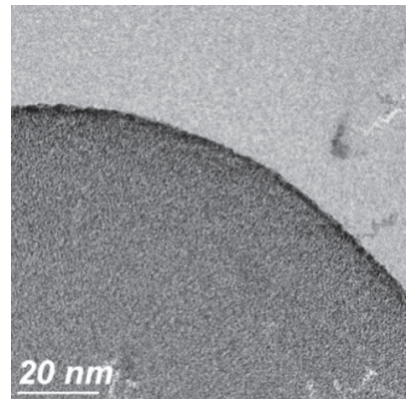

(d)

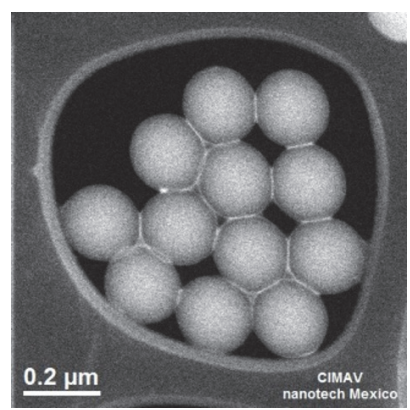

(h)

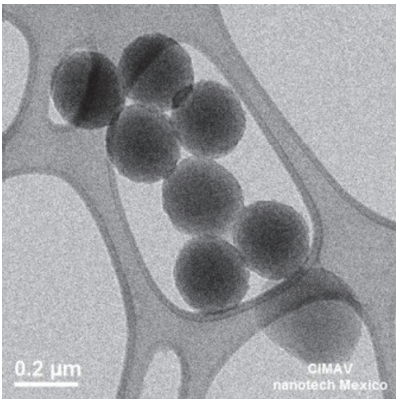

(j)

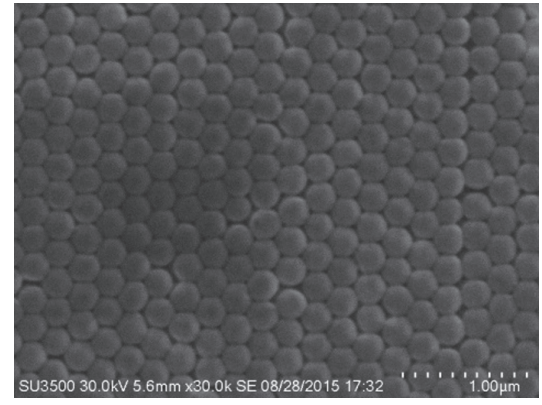

(k)

Figure 3: Bright field TEM micrograph of synthetized microspheres: Rx-24, $18 \%$ of IOA (a, b); Rx-30 with $22 \%$ of EHA (c, d); Rx-37 with $18 \%$ of HA (e, f); Rx-62 with $16 \%$ of BA (g, h); Rx-27 and Rx-53 with 13 and $18 \%$ of IBA (i, j), respectively (images from (e) to (j) were taken by STEM mode). SEM micrograph of Rx-29 with $21 \%$ of EHA (k).

the viscosity during polymerization process was responsible for the formation of an uneven surface structure. Figure 3(k) shows SEM image for film over glass microscope slide; this image shows continues distribution with all microspheres in good arrange on the substrate. Moreover, as shown in Table 2, $T_{g}$ for the $\mathrm{Rx}-29$ is $53.2^{\circ} \mathrm{C}$ and any coalescence was not observed in the microspheres that may affect the resulting opalescent films.

Figure 4 shows a SEM image of microspheres with 39\% of IOA. Well-defined particles were observed as well as the presence of particles having coalescence. The films prepared with these particles were observed to be transparent to the visible light.

3.5. Self-Assembly of Microspheres to Fabricate Photonic Films. The relationship between the size of microspheres in the films and the wavelength of maximum reflection was correlated by Bragg's law to estimate the particle diameter [15]. Thus, the calculated diameters of films with microspheres of 255 (Rx-42), 250 (Rx-29), 252 (Rx-69), 260 (Rx-56), and 255 (Rx61) $\mathrm{nm}$ are $220,226,238,241$, and $220 \mathrm{~nm}$, respectively (see Table 3).

Figure 5 shows the reflectance spectra of the films fabricated from microspheres having very similar size: 250 (Rx-29), 252 (Rx-69), 255 (Rx-42), 255 (Rx-61), and 260 $(\mathrm{Rx}-56) \mathrm{nm}$ (see Table 3). The corresponding reflection peak positions were at $528,554,512,555$, and $565 \mathrm{~nm}$, respectively. It can be observed that peaks positions in the films made up with $\mathrm{Rx}-29$ and $\mathrm{Rx}-42$ are shifted to shorter wavelengths (27 and $43 \mathrm{~nm}$ ) compared with the rest, although these particles have similar hydrodynamic diameter. These differences can be attributed to several factors: the particles are not hard spheres and can be deformed during the self-assembly due to the soft comonomer (as mentioned above, the DSC analysis 
TABLE 3: Reflection peak position of photonic crystals films, particle diameter predicted by Bragg's law, and DLS measurements.

\begin{tabular}{|c|c|c|c|c|c|}
\hline Sample ID & Comonomer & $\begin{array}{c}\text { Molar ratio } \\
\text { Sty: comonomer : } \mathrm{AA}^{\mathrm{a}}\end{array}$ & $\begin{array}{l}\text { Reflection peak } \\
(\mathrm{nm})^{\mathrm{b}}\end{array}$ & $\begin{array}{c}\text { Particle diameter } \\
\text { by Bragg's law } \\
(\mathrm{nm})^{\mathrm{c}}\end{array}$ & $D_{h}(\mathrm{~nm})^{\mathrm{d}}$ \\
\hline $\mathrm{Rx}-42$ & IOA & $73: 23: 4$ & 512 & 220 & 255 \\
\hline $\mathrm{Rx}-29$ & EHA & $67: 21: 13$ & 528 & 226 & 250 \\
\hline$R x-69$ & HA & $65: 30: 5$ & 554 & 238 & 252 \\
\hline $\mathrm{Rx}-56$ & HA & $74: 21: 5$ & 565 & 241 & 260 \\
\hline $\mathrm{Rx}-61$ & BA & $68: 18: 14$ & 555 & 220 & 255 \\
\hline
\end{tabular}

${ }^{\mathrm{a}}$ Molar ratio of comonomers in the microsphere by ${ }^{1} \mathrm{H}-\mathrm{NMR}$.

${ }^{\mathrm{b}}$ Determined by reflectance spectrometer.

${ }^{\mathrm{c}}$ Determined using Bragg's equation [15].

${ }^{\mathrm{d}}$ Determined by DLS.

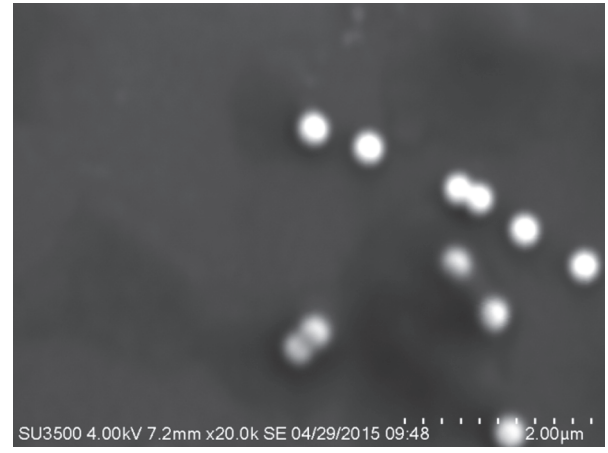

FIGURE 4: SEM micrograph of synthetized microspheres containing $39 \%$ molar amount of IOA (Rx-66, entry 4 of Table 1$)$.

shows only one $T_{g}$ ); the refractive index contrast [25-29] (e.g., type and ratio of comonomer in the microsphere and the air); defects in films caused during self-assembly; cracks and vacancies [28], and so forth.

To achieve a better understanding of these results, the positions of the reflection peak of photonic films reported by other working groups were analyzed (see Table 4).

Photonic films made with microspheres of the same size and chemical composition may exhibit a reflection peak slightly shifted. As can be seen from Table 4, microspheres of poly(Sty) having a diameter of $250 \mathrm{~nm}$ have shown a reflection peak at 525 [26] and 618 [27] nm, respectively. A small proportion of methacrylic acid was used in the formulation reported by Menshikova et al. [26].

On the other hand, since the particles have different soft comonomer content, the self-assembly of the films was carried out at temperatures below $40^{\circ} \mathrm{C}$. When colloidal crystal films containing BA and IBA (using the dip-coating method at $40^{\circ} \mathrm{C}$ ) were exposed at $70^{\circ} \mathrm{C}$ for $24 \mathrm{~h}$, it was observed that the iridescence was retained, for example, $\mathrm{Rx}$ 27 (13\% IBA), Rx-26 (15\% IBA), Rx-53 (18\% IBA), and all reactions carried out in this study with BA. Nevertheless, the films were brittle in contact with the tip of a pencil. By increasing the ratio of longer chain comonomers (HA or IOA), the self-assembly must be carried out at room

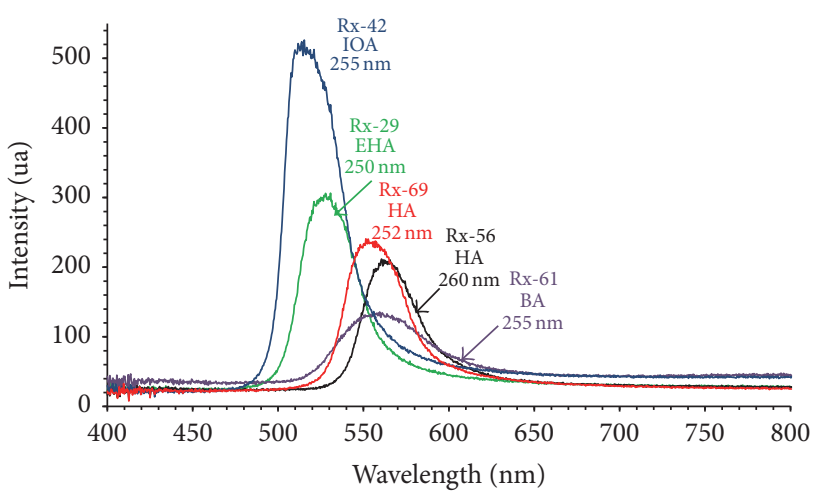

FIGURE 5: Reflectance spectra of the films made with microspheres with similar sizes. The spectra were measured at normal incidence. Sizes of microspheres were evaluated by DLS.

temperature to obtain films with good iridescence and not brittle. Moreover, all of these films are firmly attached to the substrate. Adhesive Scotch tape was placed on the surface of the films and they do not peel off.

Moreover, all the colloidal crystal films made with HA, IOA, or EHA quickly lose color when were exposed at $70^{\circ} \mathrm{C}$, including the films made with microspheres with low content of IOA or HA (e.g., Rx-24, Rx-30, and Rx-37; see Table 1). Usually in the films, the particles have a large air fraction around them, favorable to have a good optical quality, but by increasing the elastomeric fraction in the microsphere, the interstitial voids among the microspheres start coalescence; as a consequence, the air-filled spaces are reduced or disappear causing an absence of optical contrast necessary to observe the iridescence.

The films prepared with microspheres containing more than $40 \%$ of acrylates (e.g., Rx-66 and Rx-67) exhibited a lack of color with transparent appearance (see Figure S2 in Supplementary Material). The films less brittle and very iridescent were produced from Rx-42 (23\% IOA), Rx-30 (22\% EHA), Rx-57 (21\% HA), Rx-44 (25\% IBA), and Rx-35 (20\% BA). 
TABLE 4: Reflection peak position from photonic films made from polymeric microspheres having similar sizes reported in the literature.

\begin{tabular}{lccc}
\hline Reference & Microsphere $^{\mathrm{a}}$ & Size $(\mathrm{nm})^{\mathrm{b}}$ & Peak reflection (nm) \\
\hline$[9]$ & Poly(Sty-co-MMA-co-AA) & 244 & 598 \\
{$[25]$} & core-shell & 248 & 525 \\
{$[25]$} & Poly(MMA) crosslinked & 235 & 565 \\
{$[17]$} & Poly(MMA) noncrosslinked & 240 & 554 \\
{$[26]$} & Poly(Sty) & 250 & 525 \\
{$[27]$} & Poly(Sty) & 250 & 618 \\
\hline
\end{tabular}

${ }^{\mathrm{a}}$ Composition of the microsphere.

${ }^{\mathrm{b}}$ Determined by DLS.

\section{Conclusions}

Monodisperse polystyrene microspheres have been prepared by emulsion copolymerization using hexyl acrylate (HA), ethylhexyl acrylate (EHA), isooctyl acrylate (IOA), butyl acrylate (BA), or isobutyl acrylate (IBA) as comonomers. It was demonstrated that the incorporation of acrylates in the composition of the microsphere is much higher than in the formulation. Particles with a polystyrene content of $65-78 \%$ were prepared. TEM images of microspheres containing 18 to $22 \%$ molar amount of comonomer show uniform smooth surface, although microspheres with uneven surface contour were also observed. SEM image of microspheres containing $39 \%$ molar amount of IOA shows well-defined particles having coalescence. DSC shows only the presence of one $T_{g}$ in the microspheres analyzed. Consequently, these particles do not present the core-shell structure which is indicative when two or more transition phases are detected.

Films prepared with microspheres containing more than $40 \%$ of acrylates exhibit a lack of color with transparent appearance. Microspheres containing comonomer between 21 and $25 \%$ produced the less brittle films showing very iridescent colors. The photonic films prepared with long chain comonomers (HA or IOA) were especially sensitive when exposed to temperatures above $30^{\circ} \mathrm{C}$, causing decrease or disappearance of iridescence. On the contrary, some films prepared with $\mathrm{BA}$ or IBA may be subjected to $70^{\circ} \mathrm{C}$ for $24 \mathrm{~h}$ without affecting its iridescence. Negative zeta potential values $(-25$ to $-56.4 \mathrm{mV})$ indicated the presence of microspheres with high colloidal stability. No substantial variation in zeta potential was noted when the concentration of the AA was increased slightly. Finally, using hexyl, ethylhexyl, and isooctyl acrylate as comonomers enhance the quality of the films that are firmly attached to the substrate due to their adhesive properties.

\section{Competing Interests}

The authors declare no conflicts of interest.

\section{Acknowledgments}

This investigation was supported by the Dirección General de Educación Superior Tecnológica (DGEST Grant 5619.15 P), National Council of Science and Technology of México
(CONACYT) through Grant SEP-CONACYT (CB-2014-01237795), and Programa de Mejoramiento del Profesorado (PROMEP Grant 103.5-13-6605). The authors appreciate the support of J. M. Herrera and H. F Alonso from the Center of Nanoscience and Nanotechnology of UNAM (Ensenada, México) for TEM and SEM images, respectively.

\section{References}

[1] A. C. Arsenault, D. P. Puzzo, I. Manners, and G. A. Ozin, "Photonic-crystal full-colour displays," Nature Photonics, vol. 1, no. 8, pp. 468-472, 2007.

[2] J. Zhang, Z. Sun, and B. Yang, "Self-assembly of photonic crystals from polymer colloids," Current Opinion in Colloid \& Interface Science, vol. 14, no. 2, pp. 103-114, 2009.

[3] E. Tian, J. Wang, Y. Zheng, Y. Song, L. Jiang, and D. Zhu, "Colorful humidity sensitive photonic crystal hydrogel," Journal of Materials Chemistry, vol. 18, no. 10, pp. 1116-1122, 2008.

[4] P. A. Steward, J. Hearn, and M. C. Wilkinson, "Overview of polymer latex film formation and properties," Advances in Colloid and Interface Science, vol. 86, no. 3, pp. 195-267, 2000.

[5] Y.-W. Chung, I.-C. Leu, J.-H. Lee, and M.-H. Hon, "Influence of humidity on the fabrication of high-quality colloidal crystals via a capillary-enhanced process," Langmuir, vol. 22, no. 14, pp. 6454-6460, 2006.

[6] M. H. Kim, S. H. Im, and O. O. Park, "Rapid fabrication of two- and three-dimensional colloidal crystal films via confined convective assembly," Advanced Functional Materials, vol. 15, no. 8, pp. 1329-1335, 2005.

[7] X. Chen, Z. Q. Sun, Z. M. Chen, W. J. Shang, K. Zhang, and B. Yang, "Alternative preparation and morphologies of self-assembled colloidal crystals via combining capillarity and vertical deposition between two desired substrates," Colloids and Surfaces A: Physicochemical and Engineering Aspects, vol. 315, no. 1-3, pp. 89-97, 2008.

[8] J. G. McGrath, R. D. Bock, J. M. Cathcart, and L. A. Lyon, "Selfassembly of 'paint-on' colloidal crystals using poly(styrene-co$\mathrm{N}$-isopropylacrylamide) spheres," Chemistry of Materials, vol. 19, no. 7, pp. 1584-1591, 2007.

[9] J. Wang, Y. Wen, H. Ge et al., "Simple fabrication of full color colloidal crystal films with tough mechanical strength," Macromolecular Chemistry and Physics, vol. 207, no. 6, pp. 596604, 2006.

[10] B. Viel, T. Ruhl, and G. P. Hellmann, "Reversible deformation of opal elastomers," Chemistry of Materials, vol. 19, no. 23, pp. 5673-5679, 2007. 
[11] J. L. Keddie, "Film formation of latex," Materials Science and Engineering R: Reports, vol. 21, no. 3, pp. 101-170, 1997.

[12] B. You, N. Wen, L. Shi, L. Wu, and J. Zi, "Facile fabrication of a three-dimensional colloidal crystal film with large-area and robust mechanical properties," Journal of Materials Chemistry, vol. 19, no. 22, pp. 3594-3597, 2009.

[13] S. Zhang, J. Chen, and M. Taha, "Synthesis of monodisperse styrene/methyl methacrylate/acrylic acid latex using surfactant-free emulsion copolymerization in air," Journal of Applied Polymer Science, vol. 114, no. 3, pp. 1598-1605, 2009.

[14] K. Kang, C. Kan, Y. Du, and D. Liu, "Synthesis and properties of soap-free poly(methyl methacrylate-ethyl acrylate-methacrylic acid) latex particles prepared by seeded emulsion polymerization," European Polymer Journal, vol. 41, no. 3, pp. 439-445, 2005.

[15] S. H. Kim, W. K. Son, Y. J. Kim et al., "Synthesis of polystyrene/ poly(butyl acrylate) core-shell latex and its surface morphology," Journal of Applied Polymer Science, vol. 88, no. 3, pp. 595601, 2003.

[16] F. Hoshino, H. Kawaguchi, and Y. Ohtsuka, "N-substituted acrylamide-styrene copolymer lattices III. Morphology of latex particles," Polymer Journal, vol. 19, no. 10, pp. 1157-1164, 1987.

[17] S. Kim, Y. G. Seo, Y. Cho, J. Shin, S. C. Gil, and W.-M. Lee, "Optimization of emulsion polymerization for submicron-sized polymer colloids towards tunable synthetic opals," Bulletin of the Korean Chemical Society, vol. 31, no. 7, pp. 1891-1896, 2010.

[18] T. Zhao, Y. Zhang, J. Wang, Y. Song, and L. Jiang, "Fabrication of phase-change polymer colloidal photonic crystals," Journal of Nanomaterials, vol. 2014, Article ID 702089, 7 pages, 2014.

[19] C. Sun, Y. Yao, and Z. Gu, "Fabrication of elastic colloidal crystal films from pure soft spheres," Colloids and Surfaces A: Physicochemical and Engineering Aspects, vol. 402, pp. 102-107, 2012.

[20] M. Okubo, T. Fujiwara, and A. Yamaguchi, "Morphology of anomalous polystyrene/polybutyl acrylate composite particles produced by seeded emulsion polymerization," Colloid and Polymer Science, vol. 276, no. 2, pp. 186-189, 1998.

[21] M. Yoldi, C. Arcos, B.-R. Paulke, R. Sirera, W. GonzálezViñas, and E. Görnitz, "On the parameters influencing the deposition of polystyrene colloidal crystals," Materials Science and Engineering C, vol. 28, no. 7, pp. 1038-1043, 2008.

[22] C. Gaillard, G. Fuchs, C. J. G. Plummer, and P. A. Stadelmann, "The morphology of submicronsized core-shell latex particles: an electron microscopy study," Micron, vol. 38, no. 5, pp. 522$535,2007$.

[23] H. Ismail, Z. Ahmad, and F. W. Yew, "Effect of monomer composition on adhesive performance for waterborne acrylic pressure-sensitive adhesives," Journal of Physical Science, vol. 22, no. 2, pp. 51-63, 2011.

[24] O. El-Gholabzouri, M. Á. Cabrerizo-Vílchez, and R. HidalgoÁlvarez, "Zeta-potential of polystyrene latex determined using different electrokinetic techniques in binary liquid mixtures," Colloids and Surfaces A: Physicochemical and Engineering Aspects, vol. 291, no. 1-3, pp. 30-37, 2006.

[25] Z.-Z. Gu, H. Chen, S. Zhang, L. Sun, Z. Xie, and Y. Ge, "Rapid synthesis of monodisperse polymer spheres for self-assembled photonic crystals," Colloids and Surfaces A: Physicochemical and Engineering Aspects, vol. 302, no. 1-3, pp. 312-319, 2007.

[26] A. Y. Menshikova, B. M. Shabsels, N. N. Shevchenko et al., "Surface modified latex particles: synthesis and self-assembling into photonic crystals," Colloids and Surfaces A: Physicochemical and Engineering Aspects, vol. 298, no. 1-2, pp. 27-33, 2007.
[27] H. Ge, Y. Song, L. Jiang, and D. Zhu, “One-step preparation of polystyrene colloidal crystal films with structural colors and high hydrophobicity," Thin Solid Films, vol. 515, no. 4, pp. 15391543, 2006.

[28] A. Mocanu, E. Rusen, and A. Diacon, "Optical properties of the self-assembling polymeric colloidal systems," International Journal of Polymer Science, vol. 2013, Article ID 238567, 11 pages, 2013.

[29] J. Park, J. Moon, H. Shin, D. Wang, and M. Park, "Direct-write fabrication of colloidal photonic crystal microarrays by ink-jet printing," Journal of Colloid and Interface Science, vol. 298, no. 2, pp. 713-719, 2006. 

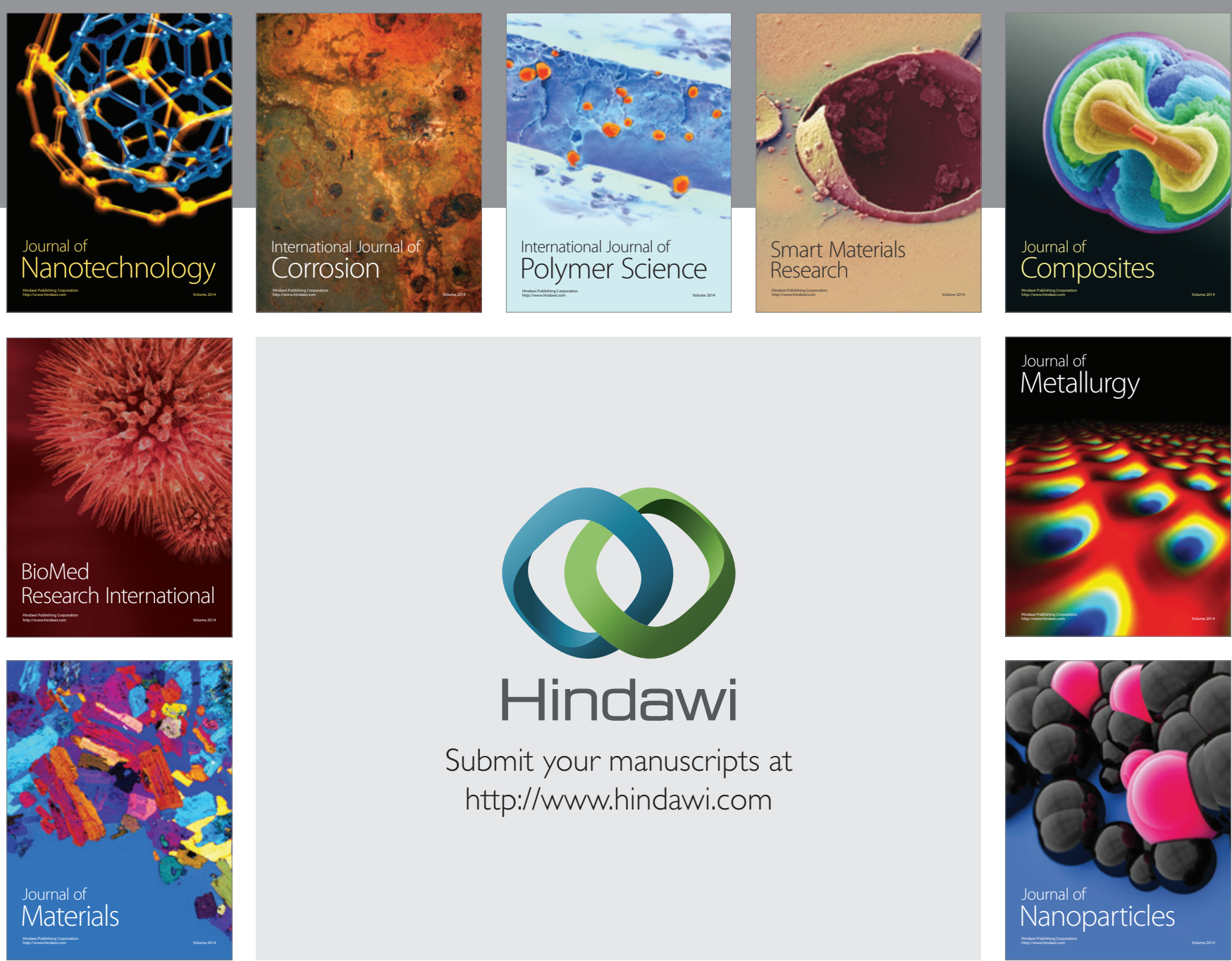

\section{Hindawi}

Submit your manuscripts at

http://www.hindawi.com

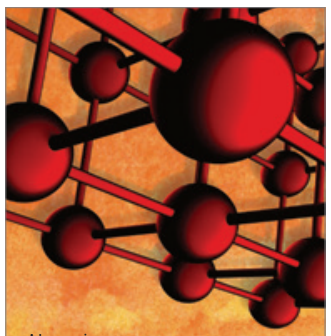

Materials Science and Engineering
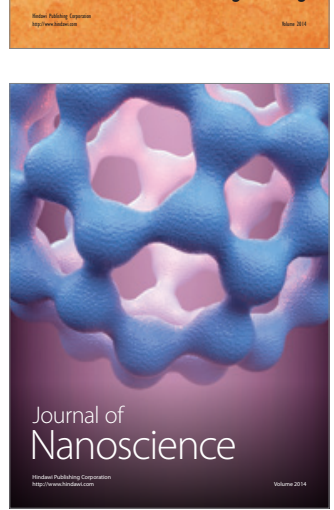
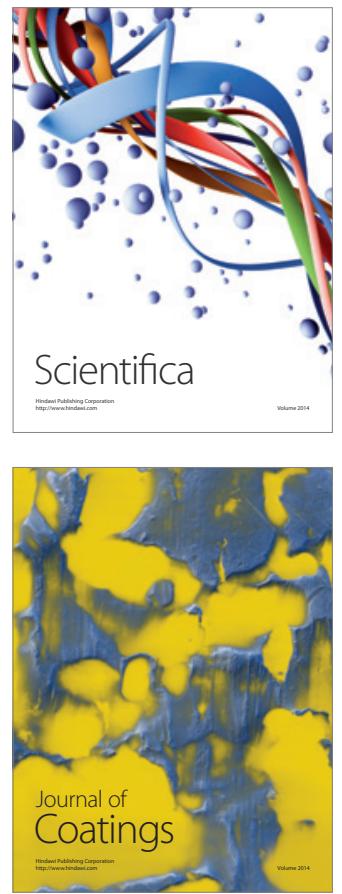
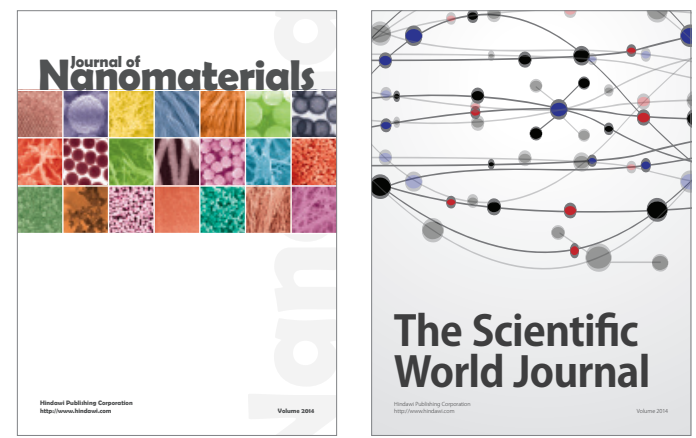

The Scientific World Journal
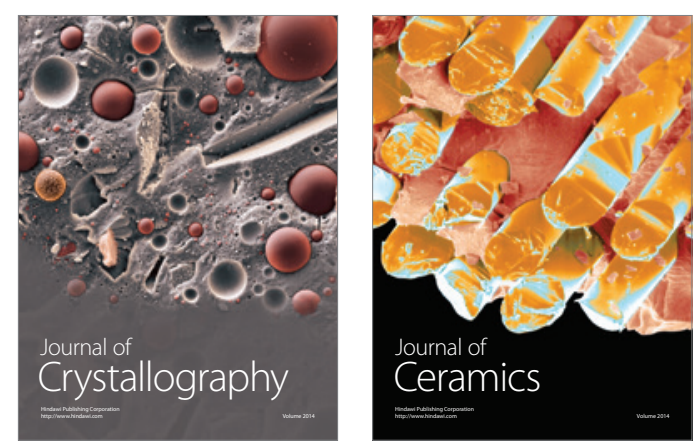
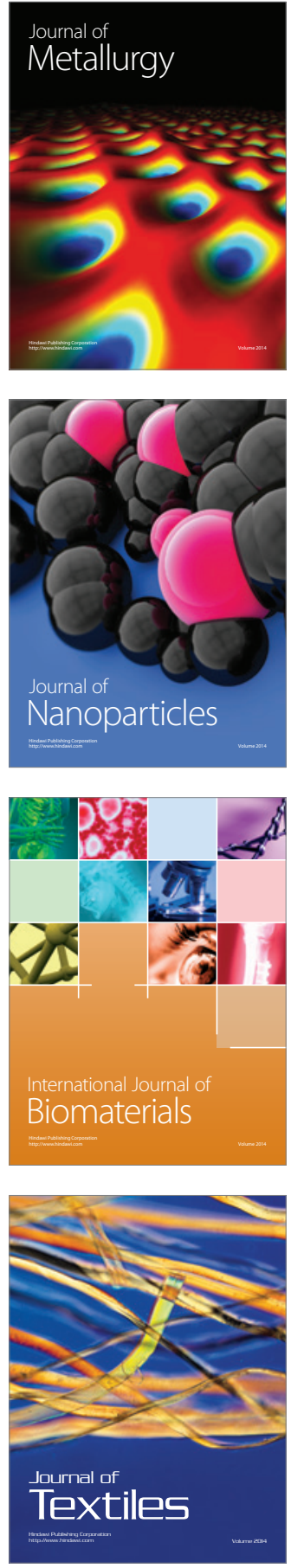